\title{
Effect of deep-frying at different temperature and time on sulfonamide residues in chicken meat-balls.
}

\begin{abstract}
Sulfonamide residues are proven to be reduced in raw chicken meat after heat treatment but in processed chicken balls it is yet to be done. The objective of the study was to determine the optimum heat treatment condition for the reduction of sulfonamide residues in production of acceptable quality chicken balls. Three heat treatment processes; boiling, deep-frying and microwaving were consecutively applied on chicken balls pre-fortified at raw stage with four types of sulfonamides(SAs) namely sulfadiazine (SDZ), sulfamethazine (SMZ), sulfamethoxazole (SMX)and sulfaquinoxaline (SQX). Boiling process was carried out at 3,6 and 9 min with temperature of 80,90 and $100{ }^{\circ} \mathrm{C}$. Boiling of chicken balls at $100{ }^{\circ} \mathrm{C}$ for 6 min was found to be the most acceptable in appearance and were brought to deep fryingprocess with temperature of 170,180 , and $190{ }^{\circ} \mathrm{C}$ and time of 3,6 , and 9 min. For deep-fried chicken balls, temperature of $180{ }^{\circ} \mathrm{C}$ for 6 min was found to be themost acceptable in appearance and was used for microwaving process at power of 100, 250, and $440 \mathrm{~W}$ for 20,40 , and $60 \mathrm{~s}$. For boiling, both factors (time andtemperature) had significant reduction for all SAs ( $<<0.05$ ). The same went for deep-frying process; time and temperature had caused significant reduction for all SAs ( $\mathrm{p}<0.05$ ). For microwave process, both factors (time and power) also showed significant reduction for all SAs $(\mathrm{p}<0.05)$. SDZ was reduced up to $62 \%$ after application of the three cooking methods continuously. For SMZ the reduction was 52\%. Meanwhile, $62 \%$ and 55\% of reduction were observed for SMX and SQX respectively.The sequence of reduction on SAs residues for all cooking methods was deep-frying > boiling > microwave. The results would be guidance for industries and consumers to reduce risk of sulfonamide residues by heat treatments.
\end{abstract}

Keyword: Sulfonamide; Deep-frying; Chicken meat balls. 